\title{
Editorial
}

\section{Globalization and community nursing}

Images are a powerful medium of communication. For participants attending the International Conference in Community Nursing Research on the 12 September 2001, images from the previous 24 hours of loss and human suffering were a poignant reminder of the challenges facing citizens across the world. Yet despite the unfolding tragedy, these shared experiences also brought a sense of unity to the professionals attending the conference.

Until that moment, globalization for many of us was merely a theoretical concept largely divorced from health care. Today however in its broadest sense globalization is no longer just concerned with international industry but is integral to health and welfare systems in every country. McGrew et al. (1992) describe globalization as 'the process by which events, decisions and activities in one part of the world can come to have significant consequences for individuals and communities in quite distant parts of the world'.

If we apply this definition to health care we can immediately start to see examples of globalization in action. Firstly, we have experienced the ease by which emerging disease in one country can quickly have consequences across the world. HIV is just one example of the reality of living in an age where geographical boundaries are no longer a barrier for the spread of disease. Secondly, globalization is apparent in the movement of the nursing workforce where recent trends have seen increasing migration of nurses. Whilst the value of this in terms of the blending of cultural backgrounds and shared professional experience is recognized, the reality may be that one country's recruitment solution creates a greater deficit in another nation.

These examples illustrate how globalization is now a part of contemporary health care. The challenge, however, is to use globalization in a positive way to advance community nursing. Implicit to this advancement is creating a more globally aware nursing climate.

Community nurses are frontline workers in delivering primary care. In many countries these nurses are the first point of health contact for the community. They are professionals with the advanced skills and knowledge to deal with a range of health problems, and someone who has a real understanding about the community and the issues that are important to the people who live there. The increasing proportion of elderly people, rising number of people affected by chronic diseases and resurgence of existing infectious diseases present a global challenge for us to develop a culture of proactive health investment, and disease prevention.

At an international level, community nurses are also facing a constant challenge of having to adapt and respond to a very changing world where threats to health are not just the result of disease but of environmental hazards, war and poverty. This changing context means that our knowledge base must also develop and grow in order for us to continue to deliver care which is up to date, and evidence based. But how do we ensure that community nurses at every level have the skills and knowledge to develop evidence-based policies and practices? Whilst practice underpinned by research is familiar to many nurses, in some countries practitioners may be at a different level of competency and readiness. Therefore a key starting point is ensuring that all practitioners are equipped with the skills which will enable them to understand, critique and utilize research in a meaningful way. By using this approach the community nurse can apply these skills to their own unique national and clinical context.

Research and education are both tools which can be used to enable evidence-based practice to flourish. However, it is the added value of researchers, practitioners and educationalists working together which truly elicits real and meaningful change in community nursing. It is the cyclical approach where research informs practice, and where prac- 
tice influences the direction of research and education which facilitates true partnership working.

Creating a knowledge base for community nursing is essential for future development and must be seen as a dynamic and on-going activity. Key players like the World Health Organization and the International Council for Nurses are instrumental in providing a global forum for shared discussion. Likewise, International Conferences in Community Healthcare Nursing Research (ICCHNR) provides an opportunity for community practitioners, researchers and educationalists to come together to share and disseminate research. Professionally, it is important for countries to have a united voice through groups like these in order to push forward the research agenda both nationally and internationally.

Partnership and collaboration need to be at the heart of intercountry working along with a shared goal to create a research active learning environment to support on-going practice development. This recognizes the importance of each country having a network of people with different types of expertise - community nurses with skills of research awareness and understanding who are key to disseminating and utilising evidence in practice settings; educationalists who can facilitate learning of research methods, and enthuse and motivate students at different levels; and researchers who can initiate and lead projects. Key to this is acknowl- edging that all these groups are essential for creating a dynamic research culture.

For me, the real essence of ICCHNR is about providing an opportunity for nurses to come together from different cultural and knowledge backgrounds who have the common bond of a shared passion for advancing community nursing. This is about leading and not being led by globalization. Mo Im Kim, a senior nurse from South Korea, said of nursing 'There is nothing around us to reverse the direction of development and change ... thus we are posed with the question of being the master of these changes or their servant'.

Now is the time to grasp the opportunity that globalization is offering, by creating an international environment of shared learning where the combined expertise of practitioners, researchers and educationalists is the framework for future community nursing advancement.

Lesley A. Whyte School of Nursing, Midwifery and Community Health/WHO Europe Collaborating Centre Glasgow Caledonian University, Glasgow, UK

\section{Reference}

McGrew, A.G. and Lewis, P.G. 1992: Global politics. Globalisation and the nation-state. Cambridge: Polity, p. 23. 